\title{
High Serum Creatine Kinase Levels in Infliximab and Vedolizumab-Treated Inflammatory Bowel Disease Patients
}

\author{
Manuel Sutter ${ }^{a}$ Petr Hruz ${ }^{b}$ Jan Hendrik Niess ${ }^{a}$ b \\ ${ }^{a}$ Department of Biomedicine, University of Basel, Basel, Switzerland; ${ }^{b}$ Clarunis - University Center for \\ Gastrointestinal and Liver Diseases, St. Clara Hospital and University Hospital of Basel, Basel, Switzerland
}

\section{Keywords}

Inflammatory bowel disease · Ulcerative colitis · Crohn's disease $\cdot$ Infliximab $\cdot$ Vedolizumab $\cdot$ Creatine kinase

\begin{abstract}
Background: TNF inhibitors are relatively safe drugs, but asymptomatic infliximab-induced high serum creatine kinase (CK) levels have been reported in $>30 \%$ of patients with inflammatory bowel disease (IBD). Whether high serum CK levels are a specific effect of treatment with TNF inhibitors has not been studied in detail. CK levels were therefore compared between infliximab- and vedolizumab-treated IBD patients. Methods: In this retrospective, monocentric study, 131 IBD cases (82 with Crohn's disease (CD), 49 with ulcerative colitis) of the Basel University Hospital IBD cohort treated either with infliximab or vedolizumab were included. Serum samples for measuring $\mathrm{CK}$, lactate dehydrogenase (LDH), C-reactive protein (CRP), and fecal calprotectin (FCal) levels were collected longitudinally and analyzed using mixed additive models. Results: No significant differences in CK levels between infliximab and vedolizumab-treated patients were observed over time. Infliximab-treated males, however, showed significantly higher CK levels than females and former smokers treated with infliximab showed significantly lower CK levels than nonsmokers. No such differences
\end{abstract}

were observed in vedolizumab-treated patients. LDH and CRP were not significantly different between infliximab- and vedolizumab-treated patients, while adjusted groups showed substantially higher LDH levels with increasing age and significantly lower LDH levels in patients with longer disease duration. Infliximab patients with CD showed significantly lower CRP. However, significantly higher FCal concentrations were noted in infliximab patients independent of diagnosis, gender, disease duration, smoking behavior, and age. Conclusion: In our cohort, high serum CK levels are not an infliximab- or vedolizumab-specific effect.

(c) 2021 The Author(s).

Published by S. Karger AG, Basel

\section{Introduction}

TNF inhibitors, such as infliximab, are effective treatments for severe inflammatory bowel disease (IBD) cases. In patients treated with TNF inhibitors, muscle-related complaints and elevated creatine kinase (CK) levels have been reported in case of reports and cross-sectional studies [1-4]. Whether elevated CK levels (high serum CK levels) in patients treated with TNF inhibitors is a specific treatment effect with TNF inhibitors, needs to be further elucidated.
C 2021 The Author(s).

Published by S. Karger AG, Basel

This article is licensed under the Creative Commons Attribution 4.0 International License (CC BY) (http://www.karger.com/Services/ OpenAccessLicense). Usage, derivative works and distribution are permitted provided that proper credit is given to the author and the original publisher.
Correspondence to:

Jan Hendrik Niess, janhendrik.niess@ unibas.ch 
Although TNF inhibitors are relatively safe drugs [58 ], one published study suggests that $>30 \%$ of IBD patients treated with infliximab have a therapy-induced, asymptomatic serum CK elevation [9]. While high serum CKlevels can occur as a side effect of various medications, such as statins [10] widely described for the treatment of hypercholesterolemia, isotretinoins [11] used for the treatment of acne and imatinib [12] prescribed for the treatment of chronic myeloid leukemia, it is not a welldocumented side effect of infliximab up to date. Moreover, dermatomyositis and polymyositis characterized by elevated CK levels can develop in IBD and rheumatoid arthritis patients treated with TNF inhibitors when paradoxical inflammation arises in these patients [1-4]. It is also unknown if high serum CK levels occur as a side effect of treatments with other biologics in IBD.

It has been suggested that the monoclonal antibody vedolizumab targeting the $\alpha_{4} \beta_{7}$ integrin heterodimer is one of the safest treatment options for IBD. The phase 3 placebo-controlled GEMINI1 and GEMINI 2 studies for the induction and maintenance of ulcerative colitis (UC) and Crohn's disease (CD) patients treated with vedolizumab reported only a few side effects, such as headache, nasopharyngitis, upper respiratory tract infections, and arthralgia $[13,14]$. In addition, the single-arm open-label phase 3 GEMINI long-term extension study reported exacerbation of UC and nasopharyngitis as the most common adverse events in patients with UC [15-17]. To our knowledge, the occurrence of therapy-induced, asymptomatic high serum CK levels has not yet been investigated in detail.

If therapy-induced, asymptomatic high serum CK levels in IBD patients depend on infliximab or vedolizumab used to treat IBD, this longitudinal study examined differences between CK levels in IBD patients treated with infliximab or vedolizumab in IBD patients treated at the University Hospital of Basel.

\section{Methods/Statistical Analysis}

\section{Study Design, Participants, and Samples}

Between January and December 2018, we included 127 IBD patients of the Basel University Hospital treated with either infliximab or vedolizumab. Inclusion criteria were as follows: (1) at least one treatment with intravenous infliximab (Remicade ${ }^{\circledR}$ and/or Inflectra ${ }^{\circledR}$ ) and/or vedolizumab (Entyvio ${ }^{\circledR}$ ) in the year 2018; (2) available CK value before the first infusion of infliximab or vedolizumab. Exclusion criteria: (1) nonspecific IBD diagnosis; (2) known polymyositis and/or rheumatoid arthritis or occurrence of polymyositis during the study period. We considered polymyositis when proximal muscle weakness was observed in the clinical exam, and persistent C-reactive protein (CRP) or erythrocyte sedimentation rate elevation was noted. Auto-antibodies (polymyositis panel), including antinuclear antibodies, anti-Jo1 antibodies, anti-Mi2 antibodies, and anti-Pm/Scl antibodies, further indicated possible polymyositis. All patients fulfilling these criteria have been excluded from the analysis. Further exclusion criteria were (3) manifestation of thyroid disease, (4) no treatment with biologics in 2018, and (5) missing CK values before the first treatment. A total of 127 patients were included in this study. This study protocol has been approved by the Ethic Commission Northwest and Central Switzerland (submission \#2019-00641).

Serum samples for measuring $\mathrm{CK}$, lactate dehydrogenase (LDH), CRP and fecal samples for calprotectin (FCal) values were collected dating back to the first treatment series at the University Hospital of Basel. All blood samples were routinely collected on each treatment day before receiving infliximab or vedolizumab and analyzed by the Clinical Laboratory, University Hospital of Basel. Briefly, CK and LDH values have been determined with UV tests (Roche) and measured with a Roche/Hitachi Cobas c system. Cutoff levels were defined according to the information provided by the manufacturer (CK: males; CK $<190$ U/L, females; CK $<170$ U/L; LDH: males; LDH 135-225 U/L, females; LDH 135-214 U/L; Roche). In this ex-post facto study design, no systematic interviews regarding physical activity were available. Fecal samples were collected intermittently and therefore did not correspond to the same timeline as the blood samples. Demographic data and medication, where available, were culled from the patient files prior to the collection of laboratory values. Because the information regarding the current medication was incomplete and inconsistent, we decided not to consider the possible effects of medications in our analysis.

\section{Statistical Analysis}

We collected the CK and LDH levels detected at every time point when the patient received infliximab or vedolizumab. CK and $\mathrm{LDH}$ values have been excluded when $>1,000 \mathrm{U} / \mathrm{L}$ and $\mathrm{LDH}$ $>500 \mathrm{U} / \mathrm{L}$ at one data point of the longitudinal data set when all other data points were below. All other data points per patients were included in the analysis to omit undesired falsifications in our regression analysis. Consequently, $99.5 \%$ of the CK and LDH values were included in the study analysis following the exclusion of $\mathrm{CK}(>1,000 \mathrm{U} / \mathrm{L})$ and $\mathrm{LDH}(>500 \mathrm{U} / \mathrm{L})$ values. Moreover, CRP values quantified as $0 \mathrm{mg} / \mathrm{L}$ was set to $0.1 \mathrm{mg} / \mathrm{L}$ to enable logarithmic transformation. Then, values beyond or below the laboratory threshold (i.e., >, <) were assigned to the threshold value to simplify analysis. Hemolytic blood samples were set to missing LDH values. All 4 clinical parameters $\mathrm{CK}, \mathrm{CRP}, \mathrm{LDH}$, and FCal, were log-transformed to receive approximately Gaussian distribution verified by quantile comparison plots. To predict the clinical parameters during the treatment with infliximab or vedolizumab, generalized additive mixed models were performed. The proper function of the time-dependent behavior was based on the principle of restricted maximum likelihood. All regression models were adjusted for age, gender, smoking status, and duration of disease. The back-transformed estimates represent geometric mean ratios (GMRs) with corresponding 95\% confidence intervals and $p$ values analyzed by the statistics program " $R$ " version 3.5.1.

Results with a $p$ value $<0.05$ were considered significant. Adjustment of significance level for multiple comparisons was omitted because of the descriptive nature of the study. 
Table 1. Demographic characteristics in infliximab- and vedolizumab-treated patients

\begin{tabular}{|c|c|c|c|c|}
\hline & \multicolumn{2}{|l|}{ Infliximab } & \multicolumn{2}{|l|}{ Vedolizumab } \\
\hline & $\mathrm{CD}(n=67)$ & $\mathrm{UC}(n=32)$ & $\mathrm{CD}(n=15)$ & $\mathrm{UC}(n=17)$ \\
\hline Age [mean (SD)], years & $42.64(15.1)$ & $40.69(12.5)$ & $41.33(14.4)$ & $45.18(14.4)$ \\
\hline Gender (female) $n(\%)$ & $31(46.3)$ & $17(53.1)$ & $10(66.7)$ & $6(35.3)$ \\
\hline Gender (male) $n(\%)$ & $36(53.7)$ & $15(46.9)$ & $5(33.3)$ & $11(64.7)$ \\
\hline Weight (mean [SD]), kg & $79.0(23.2)$ & $71.2(19.0)$ & $71.4(16.6)$ & $83.3(15.7)$ \\
\hline Smoker, $n(\%)$ & $24(35.8)$ & $4(12.5)$ & $5(33.3)$ & $5(29.4)$ \\
\hline Disease duration mean (SD), years & $13.8(11.6)$ & $10.0(7.5)$ & $14.6(11.2)$ & $9.9(6.5)$ \\
\hline \multicolumn{5}{|l|}{ Montreal classification, $n(\%)$} \\
\hline \multicolumn{5}{|l|}{ Age at diagnosis, years (CD) } \\
\hline A1 $(<16$ years $)$ & $6(9.0)$ & & $3(20.0)$ & \\
\hline A2 (16-40 years) & $40(59.7)$ & & $8(53.3)$ & \\
\hline A3 $(>40$ years $)$ & $11(16.4)$ & & $2(13.3)$ & \\
\hline NA & $10(14.9)$ & & $2(13.3)$ & \\
\hline \multicolumn{5}{|l|}{ Disease location (UC) } \\
\hline E1 (proctitis) & & $3(9.4)$ & & $0(0.0)$ \\
\hline E2 (left colitis) & & $11(34.4)$ & & $8(47.1)$ \\
\hline E3 (pancolitis) & & $8(25.0)$ & & $8(47.1)$ \\
\hline $\mathrm{NA}$ & & $10(31.3)$ & & $1(5.9)$ \\
\hline \multicolumn{5}{|l|}{ Disease location (CD) } \\
\hline L1 (terminal ileum) & $14(20.9)$ & & $4(26.7)$ & \\
\hline L2 (colon) & $12(17.9)$ & & $2(13.3)$ & \\
\hline L3 (ileum-colon) & $24(35.8)$ & & $4(26.7)$ & \\
\hline L4 (upper GI) & $2(3.0)$ & & $0(0.0)$ & \\
\hline Others & $5(7.5)$ & & $3(20.0)$ & \\
\hline NA & $10(14.9)$ & & $2(13.3)$ & \\
\hline \multicolumn{5}{|l|}{ Disease behavior (CD) } \\
\hline B1 (nonstenotic, nonpenetrating) & $25(37.3)$ & & $6(40.0)$ & \\
\hline B1p & $2(3.0)$ & & $0(0.0)$ & \\
\hline B2 (stenotic) & $15(22.4)$ & & $4(26.7)$ & \\
\hline $\mathrm{B} 2 \mathrm{p}$ & $1(1.5)$ & & $1(6.7)$ & \\
\hline B3 (penetrating) & $7(10.4)$ & & $2(13.3)$ & \\
\hline B3p (perianal penetrating) & $5(7.5)$ & & $0(0.0)$ & \\
\hline Others & $2(3.0)$ & & $0(0.0)$ & \\
\hline NA & $10(14.9)$ & & $2(13.3)$ & \\
\hline \multicolumn{5}{|c|}{ Use of other medication during biological treatment, $n(\%)$} \\
\hline Beta-blockers & $7(10.4)$ & $2(6.3)$ & $2(13.3)$ & $2(11.8)$ \\
\hline Glucocorticoids & $17(25.4)$ & $13(40.6)$ & $3(20.0)$ & $9(52.9)$ \\
\hline Thyroxines & $3(4.5)$ & $0(0.0)$ & $0(0.0)$ & $2(11.8)$ \\
\hline Statine & $6(9.0)$ & $0(0.0)$ & $1(6.7)$ & $3(17.6)$ \\
\hline Methotrexat & $4(6.0)$ & $3(9.4)$ & $1(6.7)$ & $0(0.0)$ \\
\hline 5-Aminosalicylic acid & $12(17.9)$ & $4(12.5)$ & $1(6.7)$ & $1(5.9)$ \\
\hline \multicolumn{5}{|l|}{ IBD surgery, $n(\%)$} \\
\hline Yes & $31(46.3)$ & $2(6.3)$ & $8(53.3)$ & $2(11.8)$ \\
\hline \multicolumn{5}{|l|}{ Disease activity, $n(\%)$} \\
\hline $\mathrm{CRP}>5 \mathrm{mg} / \mathrm{L}$ & $57(85.1)$ & $22(68.8)$ & $10(66.7)$ & $16(94.1)$ \\
\hline $\mathrm{FCal}>100 \mu \mathrm{g} / \mathrm{g}$ & $48(71.6)$ & $20(62.5)$ & $6(40.0)$ & $8(47.1)$ \\
\hline
\end{tabular}

$\mathrm{CK}$, creatine kinase; LDH, lactate dehydrogenase; CRP, C-reactive protein; FCal, fecal calprotectin; CD, Crohn's disease; UC, ulcerative colitis, IBD, inflammatory bowel disease; SD, standard deviation.

\section{Results}

Demographic Characteristics and CK Concentrations Of the 127 considered individuals, 99 patients were treated with infliximab (88 with Remicade ${ }^{\circledR}$ and 11 with
Inflectra $\left.{ }^{\circledR}\right)$, and 32 patients were treated with vedolizumab (Table 1). Four out of the 32 vedolizumab-patients, who initially were treated with infliximab, changed to vedolizumab-treatment during the study period and therefore appeared in both treatment groups, leading to a total 


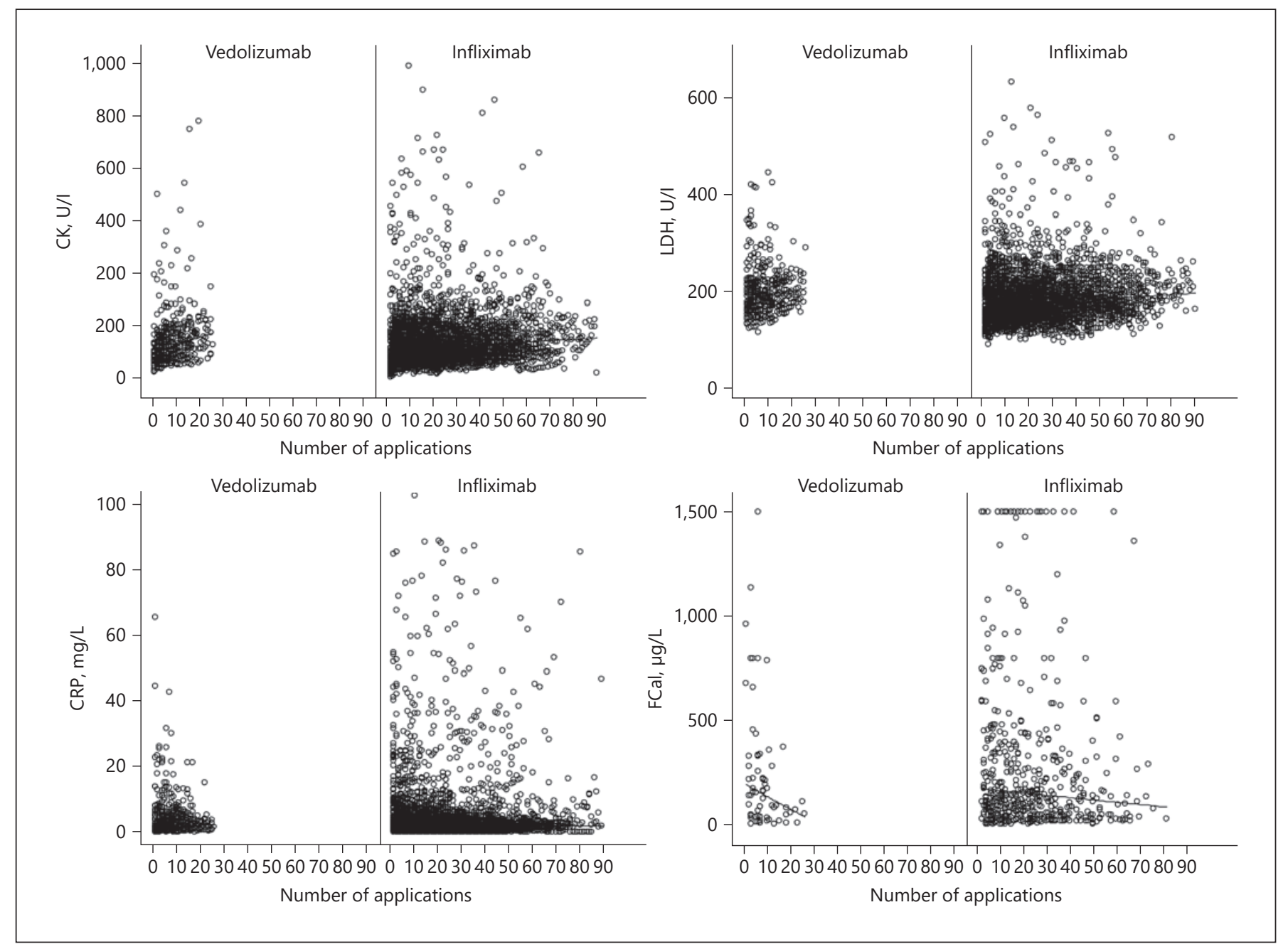

Fig. 1. Serum CK, LDH, CRP, and FCal levels in infliximab- and vedolizumab-treated patients. Serum was drawn before infliximab or vedolizumab was given. After induction therapy, every 6-8 weeks infliximab or vedolizumab was given. Values at application 1 indicated the pre-medication values since blood was taken before the biologic was given. CK, creatine kinase; LDH, lactate dehydrogenase; CRP, C-reactive protein; FCal, fecal calprotectin.

of 131 treatment cases analyzed. While only $11 \%$ of the infliximab-treated patient received Inflectra ${ }^{\circledR}$ and the remaining $89 \%$ Remicade ${ }^{\circledR}$; we argue that there will be no measurable differences in the evaluated laboratory values. Because the two medications have a similar active substance, we refer to them as infliximab. Two-thirds of the infliximab patients suffered from CD. In vedolizumabpatients, the distribution of CD and UC was about equal. In both medication groups, the sex distribution was similar. The mean age of all patient groups ranged from 40 to 45 years, and the disease duration was between 9 and 14 years. The distribution of smoking habits in the different medication groups was equally distributed with roughly
$30 \%$ smokers, $37 \%$ nonsmokers, $13 \%$ former smokers, and $20 \%$ with unknown smoking habits. We confirmed that the treatment with infliximab or vedolizumab reduced FCal concentrations in our cohort (Fig. 1). Table 2 shows the median concentration of CK, LDH, CRP, and FCal with higher CK levels in males and no profound differences between CD and UC after the beginning of the treatment. Table 3 shows the number of patients where the laboratory marker concentrations exceeded the laboratory reference range at least once. More than $50 \%$ of the patients had at least one value above the reference range. However, the mean CK was elevated in $<20 \%$ of the cases (Table 4). 
Table 2. Median concentration of biomarkers separately analyzed for CD or UC

\begin{tabular}{llccc}
\hline & & Female & Male & Total \\
\hline CD & & & \\
CK & Median (IQR) & $90(63 ; 142)$ & $106(74 ; 151)$ & $99.5(69 ; 147)$ \\
LDH & Median (IQR) & $184(161 ; 219)$ & $169(149 ; 197)$ & $176(153 ; 206)$ \\
CRP & Median (IQR) & $2(0.9 ; 4.9)$ & $2.2(1.1 ; 4.8)$ & $2.1(1 ; 4.9)$ \\
FCal & Median (IQR) & $143(62.2 ; 344.5)$ & $115.5(51 ; 234.5)$ & $126(53 ; 295)$ \\
UC & & & & \\
CK & Median (IQR) & $76(49 ; 106)$ & $139(103 ; 182.2$ & $107(69 ; 154)$ \\
LDH & Median (IQR) & $180(157 ; 209.5)$ & $196(169 ; 228)$ & $189(162 ; 221)$ \\
CRP & Median (IQR) & $2.2(0.8 ; 6.2)$ & $1.4(0.5 ; 2.9)$ & $1.7(0.6 ; 4.1)$ \\
FCal & Median (IQR) & $98(40.8 ; 685.3)$ & $178(39 ; 664)$ & $159(39 ; 679.8)$ \\
\hline
\end{tabular}

High serum CK levels defined as $>170 \mathrm{U} / \mathrm{L}$ in females and $>190 \mathrm{U} / \mathrm{L}$ in males. Elevated LDH concentrations defined as $>214 \mathrm{U} / \mathrm{L}$ in females and $>225 \mathrm{U} / \mathrm{L}$ in males. Increased CRP defined as $>10 \mathrm{mg} / \mathrm{L}$. Increased FCal defined as $>50 \mu \mathrm{g} / \mathrm{L}$. CK, creatine kinase; LDH, lactate dehydrogenase; CRP, C-reactive protein; FCal, fecal calprotectin; CD, Crohn's disease; UC, ulcerative colitis; IQR, interquartile range.

Table 3. Number of cases where laboratory values exceed the laboratory reference range at least once

\begin{tabular}{|c|c|c|c|c|}
\hline & \multicolumn{2}{|l|}{ Infliximab } & \multicolumn{2}{|l|}{ Vedolizumab } \\
\hline & $\begin{array}{l}\mathrm{CD} \\
\left(N_{\mathrm{F}}=31 ; N_{\mathrm{M}}=36\right)\end{array}$ & $\begin{array}{l}\mathrm{UC} \\
\left(N_{\mathrm{F}}=17 ; N_{\mathrm{M}}=15\right)\end{array}$ & $\begin{array}{l}\mathrm{CD} \\
\left(N_{\mathrm{F}}=10 ; N_{\mathrm{M}}=5\right)\end{array}$ & $\begin{array}{l}\mathrm{UC} \\
\left(N_{\mathrm{F}}=6 ; N_{\mathrm{M}}=1\right)\end{array}$ \\
\hline \multicolumn{5}{|l|}{ CK } \\
\hline Female $>157 \mathrm{U} / \mathrm{L}, n(\%)$ & $16(51.6)$ & $7(41.2)$ & $4(40.0)$ & $2(33.3)$ \\
\hline Male > $200 \mathrm{U} / \mathrm{L}, n(\%)$ & $25(69.4)$ & $11(73.3)$ & $2(40.0)$ & $9(81.8)$ \\
\hline \multicolumn{5}{|l|}{$\mathrm{LDH}$} \\
\hline Female $>214 \mathrm{U} / \mathrm{L}, n(\%)$ & $27(87.1)$ & $13(76.5)$ & $9(90.0)$ & $6(100)$ \\
\hline Male >225 U/L, $n(\%)$ & $30(83.3)$ & $15(100)$ & $4(80.0)$ & $7(63.6)$ \\
\hline \multicolumn{5}{|l|}{ CRP } \\
\hline$>10 \mathrm{mg} / \mathrm{L}, n(\%)$ & $46(68.7)$ & $17(53.1)$ & $9(60.0)$ & $8(47.1)$ \\
\hline \multicolumn{5}{|l|}{ FCal } \\
\hline$>50 \mu \mathrm{g} / \mathrm{L}, n(\%)$ & $54(80.6)$ & $24(75.0)$ & $7(46.7)$ & $9(52.9)$ \\
\hline
\end{tabular}

$\mathrm{CK}$, creatine kinase; LDH, lactate dehydrogenase; CRP, C-reactive protein; FCal, fecal calprotectin; CD, Crohn's disease; UC, ulcerative colitis.

CK in Infliximab- and Vedolizumab-Treated Patients

We applied a mixed additive model to our data set as we have longitudinally measured CK concentrations. This data set includes repeated CK, LDH, CRP, and FCal measures in the infliximab-treated patients every 8 weeks for 90 months and repeated CK measures in the vedolizumab group for 20 months every 8 weeks (Fig. 2). Before analysis, we excluded outliers with CK $>1,000$ $\mathrm{U} / \mathrm{L}$ and $\mathrm{LDH}>500 \mathrm{U} / \mathrm{L}$, when all other data points of the same patient have been below. We compared the GMR of infliximab and vedolizumab on CK to confirm the possibility that the previously described asymptom- atic CK elevation in infliximab-treated patients depends on TNF blockers. This analysis did not reveal significant differences in CK between infliximab- and vedolizumab-treated patients (Table 5). We also included into our analysis LDH, CRP, and FCal measures. LDH and CRP were not significantly different between infliximab-and vedolizumab-treated patients. Of note, we observed significantly lower FCal concentrations in vedolizumab than infliximab-treated patients (Table 5). We also compared the effect of the disease diagnosis (CD or UC) on CK in our cohort (Table 6). CK levels were similar between CD and UC patients. Also, similar 
Table 4. Number of cases with elevated mean CK

\begin{tabular}{|c|c|c|c|c|}
\hline & \multicolumn{2}{|l|}{ Infliximab } & \multicolumn{2}{|l|}{ Vedolizumab } \\
\hline & $\begin{array}{l}\mathrm{CD} \\
\left(N_{\mathrm{F}}=31 ; N_{\mathrm{M}}=36\right)\end{array}$ & $\begin{array}{l}\mathrm{UC} \\
\left(N_{\mathrm{F}}=17 ; N_{\mathrm{M}}=15\right)\end{array}$ & $\begin{array}{l}\mathrm{CD} \\
\left(N_{\mathrm{F}}=10 ; N_{\mathrm{M}}=5\right)\end{array}$ & $\begin{array}{l}\mathrm{UC} \\
\left(N_{\mathrm{F}}=6 ; N_{\mathrm{M}}=1\right)\end{array}$ \\
\hline \multicolumn{5}{|l|}{$\mathrm{CK}$} \\
\hline Female mean $>157 \mathrm{U} / \mathrm{L}, n(\%)$ & $4(12.9)$ & $1(5.9)$ & $2(20)$ & $0(0)$ \\
\hline CK & & & & \\
\hline Male mean $>200 \mathrm{U} / \mathrm{L}, n(\%)$ & $5(13.9)$ & $2(13.3)$ & $1(20)$ & $1(100)$ \\
\hline
\end{tabular}

CK, creatine kinase; CD, Crohn's disease; UC, ulcerative colitis.

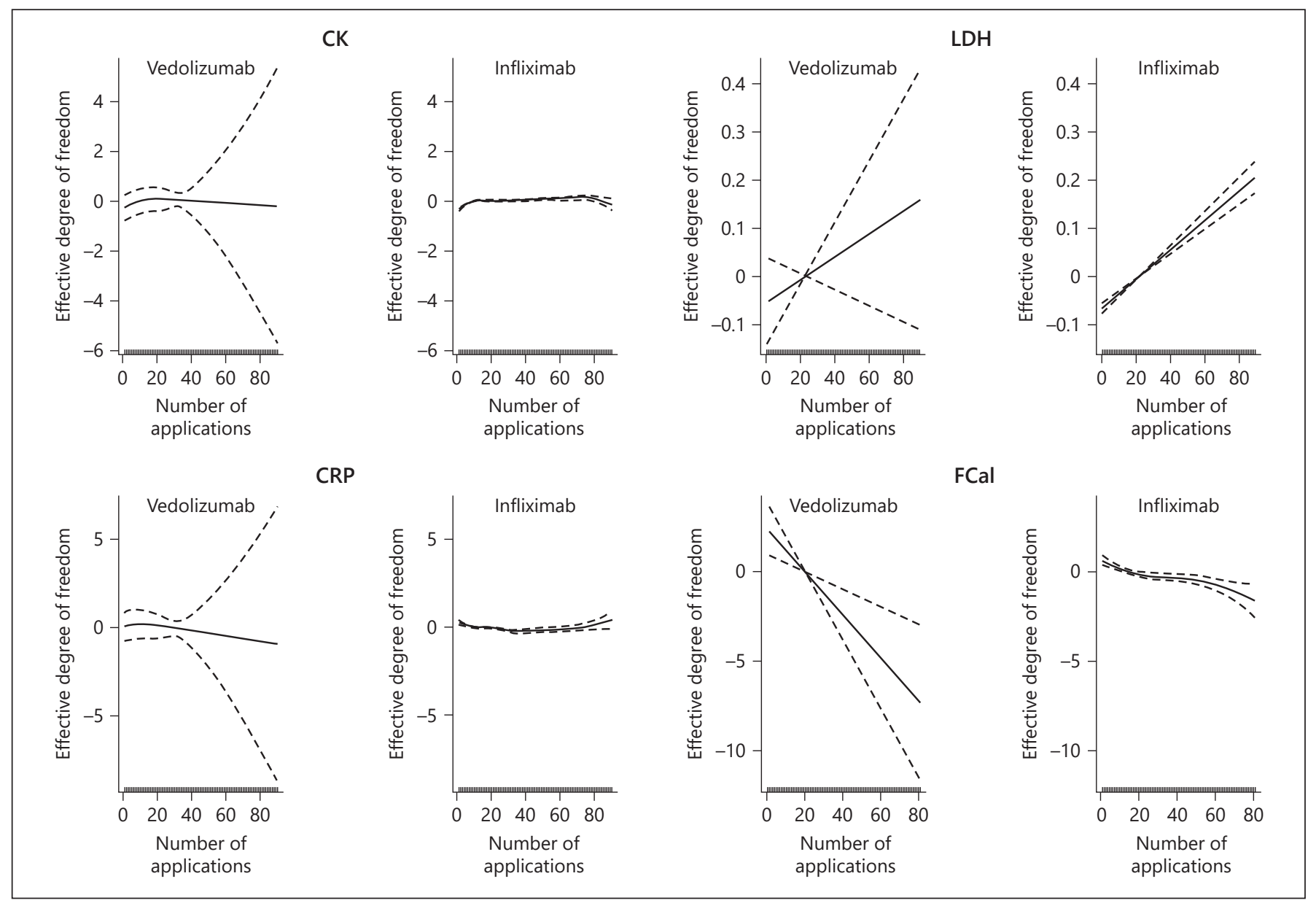

Fig. 2. Assumptions of the additive mixed error-component model for serum CK, LDH, CRP and FCal. Premedication values are indicated at application \#1 because blood was drawn before infliximab or vedolizumab was given. CK, creatine kinase; LDH, lactate dehydrogenase; CRP, C-reactive protein; FCal, fecal calprotectin.

CRP and FCal concentrations were observed in CD and UC patients, and UC patients had increased LDH concentrations (Table 6). Taken together, we did not observe significant differences in CK levels between inflix- imab or vedolizumab when the data was not adjusted for disease diagnosis, sex, age, smoking, and disease duration. 
Table 5. Comparison of serum CK, LDH, CRP, and FCal over time between medication groups

\begin{tabular}{llll}
\hline Laboratory marker & Medication/comparison & GMR $(95 \%$ CI $)$ & $p$ value \\
\hline CK & Infliximab versus vedolizumab & $0.91(0.53 ; 1.55)$ & 0.7221 \\
LDH & Infliximab versus vedolizumab & $0.93(0.84 ; 1.02)$ & 0.1216 \\
CRP & Infliximab versus vedolizumab & $0.68(0.28 ; 1.67)$ & 0.4006 \\
FCal & Infliximab versus vedolizumab & $7.28(2.22 ; 23.86)$ & 0.001 \\
\hline
\end{tabular}

CK, creatine kinase; LDH, lactate dehydrogenase; CRP, C-reactive protein; FCal, fecal calprotectin; CI, confidence interval; GMR, geometric mean ratio.

Table 6. Comparison of serum CK, LDH, CRP, and FCal over time between CD and UC

\begin{tabular}{llll}
\hline Laboratory marker & Diagnosis/comparison & GMR (95\% CI) & $p$ value \\
\hline CK & Ulcerative colitis versus Crohn's disease & $1.06(0.85 ; 1.31)$ & 0.61 \\
LDH & Ulcerative colitis versus Crohn's disease & $1.07(1.01 ; 1.14)$ & 0.046 \\
CRP & Ulcerative colitis versus Crohn's disease & $0.77(0.49 ; 1.21)$ & 0.26 \\
FCal & Ulcerative colitis versus Crohn's disease & $0.91(0.51 ; 1.61)$ & 0.75 \\
\hline
\end{tabular}

CK, creatine kinase; LDH, lactate dehydrogenase; CRP, C-reactive protein; FCal, fecal calprotectin; CI, confidence interval; GMR, geometric mean ratio; CD, Crohn's disease; UC, ulcerative colitis.

\section{Increased CK in Infliximab-Treated Patients after} Adjustment for Gender

To pursue our analysis further, we adjusted the data for disease diagnosis, sex, age, smoking, and disease duration. The adjustment of the data for sex but not for age and disease duration revealed significant differences in the GMR on CK levels for infliximab-treated patients. The ex-smoking status also correlated with increased CK levels in the infliximab-treated group (Table 7) and see online suppl. Table 1; for all online suppl. material, see www.karger.com/doi/10.1159/000518624). Furthermore, the adjustment for age and disease duration correlated with $\mathrm{LDH}$ in infliximab-treated patients, and the adjustment for disease and smoking status correlated with CRP levels (online suppl. Tables 2 and 3). The adjustment for disease diagnosis, sex, age, smoking, and disease duration did not affect FCal (online suppl. Table 4). Altogether, our data revealed only significant differences in GMR in infliximab-treated patients after adjustment.

\section{Increased CK in Males Treated with Infliximab}

We analyzed the effects of sex, age, smoking, and disease duration in infliximab- and vedolizumab-treated patients. Male status and not age correlated with increased CK levels in the infliximab group. Statistical significance was not reached in the vedolizumab group (Table 7, on- line suppl. Table 1) independent of diagnosis and medication. Males had an approximately $1.5 \times$ higher CK than females (online suppl. Table 1). In addition, age and disease duration correlated with LDH levels in the infliximab-treated patients (online suppl. Table 2). Differences in disease diagnosis, sex, age, smoking, and disease duration within infliximab- or vedolizumab-treated patients were not observed. Taken together, the data suggest that males treated with infliximab had higher CK levels associated with increased FCal levels.

\section{Discussion}

Asymptomatic high serum CK levels have been reported in infliximab-treated IBD patients [9]. In this study, we did not find a specific influence on CK levels in infliximab-treated patients, since vedolizumab-treated patients had also elevated CK levels occurred, while infliximab and vedolizumab represent different modes of action. Males treated with infliximab had significantly higher CK levels than females.

In line with our study, Theodoraki et al. [9] also reported elevated CK levels in infliximab-treated patients. More than $30 \%$ of IBD patients treated with infliximab had persistent, asymptomatic high serum CK levels [9]. 
Table 7. Comparison of serum CK over time between diagnosis, sex, age, smoking status, and disease duration within a medication group

\begin{tabular}{lllll}
\hline Parameter & $\begin{array}{l}\text { Laboratory } \\
\text { marker }\end{array}$ & Medication & GMR $(95 \%$ CI $)$ & $p$ value \\
\hline Diagnosis (CD vs. UC) & CK & Infliximab & $0.95(0.7241 ; 1.2457)$ & 0.71 \\
& & Vedolizumab & $1.20(0.8067 ; 1.7994)$ & 0.36 \\
\hline Sex (male vs. female) & CK & Infliximab & $1.59(1.2390 ; 2.0339)$ & $<0.001$ \\
& & Vedolizumab & $1.38(0.9097 ; 2.0953)$ & 0.13 \\
\hline Age, years & CK & Infliximab & $1.00(0.9878 ; 1.0062)$ & 0.52 \\
& & Vedolizumab & $1.00(0.9868 ; 1.0180)$ & 0.77 \\
\hline Smoker (yes vs. no) & CK & Infliximab & $0.88(0.6626 ; 1.1668)$ & 0.37 \\
& & Vedolizumab & $1.12(0.7336 ; 1.6997)$ & 0.61 \\
\hline Smoker (ex vs. no) & CK & Infliximab & $0.66(0.4699 ; 0.9291)$ & 0.02 \\
& & Vedolizumab & $0.82(0.4409 ; 1.5116)$ & 0.52 \\
\hline Disease duration & CK & Infliximab & $1.00(0.9879 ; 1.0134)$ & 0.93 \\
& & Vedolizumab & $1.00(0.9728 ; 1.0202)$ & 0.75 \\
\hline
\end{tabular}

CK, creatine kinase; CI, confidence interval; GMR, geometric mean ratio; CD, Crohn's disease; UC, ulcerative colitis.

We report here that $<20 \%$ of all treated cases had persistent high serum CK levels. Several reasons may contribute to the difference between our analysis and the study by Theodoraki and colleagues [9]. First, we used all available CK values, not just the last three values like in the abovementioned Greek study. Second, we did not differentiate between outpatient and hospitalized patients. Third, we considered the different reference values for females and males. Fourth, due to unavailable additional patient data, we could not exclude other causes for high serum CK levels.

Moreover, we included vedolizumab-treated patients in our analysis to receive insights into whether high serum CK levels observed in infliximab-treated IBD patients depend on anti-TNF treatment. But significant differences in CK levels between infliximab and vedolizumab-treated patients were not observed. There might be the possibility to consider that other biologic classes, such as anti-IL12p40 antibodies, may influence CK levels. Future investigations are required to test whether ustekinumab affects CK values in IBD patients.

Infliximab is a monoclonal, chimeric immunoglobulin G (IgG) antibody targeting TNF $[18,19]$, while vedolizumab is a monoclonal IgG antibody highly selectively blocking the $\alpha_{4} \beta_{7}$-integrins $[20,21]$. Infliximab neutralizes TNF, inhibits outside-inside signaling in cells expressing membrane-bound TNF, and induces apoptosis of T cells [19]. Vedolizumab binds to $\alpha_{4} \beta_{7}$-integrins reducing the migration of lymphocytes and monocytes into the intestinal tissue $[20,22]$. The different mode of action between infliximab and vedolizumab does not explain increased CK levels in IBD patients treated with biologics in our study. Since infliximab is a chimeric antibody with $25 \%$ murine and $75 \%$ human sequences [19], we initially speculated that the high percentage of murine sequences within infliximab might elevate serum CK levels observed in infliximab-treated IBD patients.

In contrast to infliximab, the immunogenicity of vedolizumab was overcome by cloning the binding site of the murine ACT- 1 antibody onto a human IgG $_{1}$ antibody $[21,22]$. Also, antibody-mediated cellular or complement cytotoxicity could contribute to high serum CK levels observed in patients treated with infliximab or vedolizumab. Of note, the fragment crystallizable region of vedolizumab is mutated to prevent cytotoxicity [21, 23]. Our study indicates that the mode of action of the respective antibody, the percentage of mouse sequences in the antibody, and antibody-mediated cellular or complement cytotoxicity do not explain the increased CK levels in IBD patients treated with infliximab or vedolizumab. At this stage, we cannot explain why asymptomatic high serum CK levels occurred in approximately $20 \%$ of patients treated with biologics. However, our findings that infliximab-treated males had higher CK levels than females 
suggest that men treated with biologics have elevated CK levels. Other studies show higher CK levels in healthy men due to more increased muscle mass [24-26], which is the possible explanation for our findings.

Outliers in CK and LDH values were excluded from the statistical analysis. Most of the excluded values were nonrecurring. While over $50 \%$ of the cases in our cohort had at least one elevated CK value, only $0.5 \%$ of all CK values superseded a value of $1,000 \mathrm{U} / \mathrm{L}$. Those cases often came along with high LDH values over $500 \mathrm{U} / \mathrm{L}$. Such a combination could suggest rhabdomyolysis or excessive physical activity [27], as anti-TNF-alpha medications are not commonly known as use for medication-induced rhabdomyolysis. Since most CRP levels were not elevated together with $\mathrm{CK}$ and $\mathrm{LDH}$, inflammatory causes are less likely. With the exclusion criteria, where patients with proximal muscle weakness have been excluded, polymyositis as a possible explanation for elevated CK values is unlikely. As well, the presence of thyroid disease was an exclusion criterion in our study. However, mild to moderate physical activity could contribute to elevated CK levels in our study. Because we collected the data in a retrospective manner, we could not systematically assess physical activity in our cohort with a specific questionnaire such as the global physical activity questionnaire. The patients with a persistent CK evaluation did not have a concomitant use of statins.

We also observed significantly lower FCal concentrations in vedolizumab-treated patients than infliximabtreated patients. As FCal concentrations correlate to the number of granulocytes in the gastrointestinal system, it is used as a marker for intestinal inflammation [28, 29]. These observations could imply that vedolizumab has a better anti-inflammatory effect than infliximab in the intestinal tract, possibly due to the higher gastrointestinal specificity of vedolizumab. In the VARSITY trial, which compared the efficacy of vedolizumab with the TNF blocker adalimumab in UC, vedolizumab-treated UC patients received higher percentages of clinical remission at week 52 [30]. Because the group size and duration of treatment in our study were unequal between the infliximab and vedolizumab group due to the later approval of vedolizumab, we do not want to overemphasize this aspect. The main goal of our study was to investigate whether treatment with infliximab or vedolizumab influences CK levels in IBD patients.

In conclusion, our study showed that high serum CK levels in IBD patients did not specifically depend on antiTNF treatment with infliximab. To determine whether vedolizumab leads to an asymptomatic and persistent high serum CK level, further prospective studies are needed regarding the possible difference between infliximab and vedolizumab in elevating CK values. High serum CK levels in IBD patients treated with biologics need to be carefully assessed and potentially life-threatening conditions ruled out before therapy with biologics is continued.

\section{Acknowledgments}

We thank Urs Simmen for statistical support and Sandra Mitrovic for discussions on measurements of CK and LDH levels and on the definitions of CK and LDH cutoff levels.

\section{Statement of Ethics}

The Ethics Commission Northwest and Central Switzerland has approved this project (submission \#2019-00641). The laboratory tests analyzed in our study are routine laboratory tests taken at every visit of the patient. The Ethics Commission Northwest and Central Switzerland has agreed that we do not need to ask for a written informed consent due to the retrospective nature of data analysis.

\section{Conflict of Interest Statement}

J.H.N. has received an unrestricted research grant from Vifor and consultancy fees from AbbVie, Jannsen, MSD, Takeda, and Pfizer. P.H. has received consultancy fees from AbbVie, Jannsen, MSD, Takeda, Pfizer, Pierre Fabre, Sandoz, and Vifor.

\section{Funding Sources}

An unrestricted research grant from Vifor supported the project. Vifor did not have any influence on the design of the study protocol and analysis of the data.

\section{Author Contributions}

M.S. acquired the data, interpreted the data, and wrote the manuscript. P.H. analyzed the data and revised the manuscript. J.H.N. designed the project, interpreted the data, and wrote the manuscript. M.S., P.H., and J.H.N. approved the final version of the manuscript.

\section{Data Availability Statement}

All data generated or analyzed during this study are included in this article and its online suppl. material files. Further inquiries can be directed to the corresponding author. 


\section{References}

1 Riolo G, Towheed TE. Anti-tumor necrosis factor inhibitor therapy-induced dermatomyositis and fasciitis. J Rheumatol. 2012; 39(1):192-4.

2 Brunasso AMG, Aberer W, Massone C. New onset of dermatomyositis/polymyositis during anti-TNF-therapies: a systematic literature review. ScientificWorldJournal. 2014; 2014:179180.

3 Takata M, Yamasaki A, Yamada N, Hagino H, Funaki Y, Harada T, et al. A case of clinically amyopathic dermatomyositis that developed during anti-TNF-a therapy for rheumatoid arthritis. Allergol Int. 2018;67(2):286-8.

4 Zengin O, Onder ME, Alkan S, Kimyon G, Hüseynova N, Demir ZH, et al. Three cases of anti-TNF induced myositis and literature review. Rev Bras Reumatol Engl Ed. 2017;57(6): 590-5.

5 Khanna R, Feagan BG. Safety of infliximab for the treatment of inflammatory bowel disease: current understanding of the potential for serious adverse events. Expert Opin Drug Saf. 2015;14(6):987-97.

6 Scheinfeld N. A comprehensive review and evaluation of the side effects of the tumor necrosis factor alpha blockers etanercept, infliximab and adalimumab. J Dermatolog Treat. 2004;15(5):280-94.

7 Zabana Y, Domènech E, Mañosa M, GarciaPlanella E, Bernal I, Cabré E, et al. Infliximab safety profile and long-term applicability in inflammatory bowel disease: 9-year experience in clinical practice. Aliment Pharmacol Ther. 2010;31(5):553-60.

8 Fidder H, Schnitzler F, Ferrante M, Noman M, Katsanos K, Segaert S, et al. Long-term safety of infliximab for the treatment of inflammatory bowel disease: a single-centre cohort study. Gut. 2009;58(4):501-8.

9 Theodoraki E, Orfanoudaki E, Foteinogiannopoulou K, Koutroubakis IE. Asymptomatic hyperCKemia during infliximab therapy in patients with inflammatory bowel disease. Inflamm Bowel Dis. 2018;24(6):1266-71.

10 Mammen AL. Statin-associated autoimmune myopathy. N Engl J Med. 2016;374(7):664-9.
11 Kaymak Y. Creatine phosphokinase values during isotretinoin treatment for acne. Int J Dermatol. 2008;47(4):398-401.

12 Gordon JK, Magid SK, Maki RG, Fleisher M, Berman E. Elevations of creatine kinase in patients treated with imatinib mesylate $\left(\right.$ Gleevec $\left.^{\mathrm{Tm}}\right)$. Leuk Res. 2010;34(6):827-9.

13 Feagan BG, Rutgeerts P, Sands BE, Hanauer S, Colombel JF, Sandborn WJ, et al. Vedolizumab as induction and maintenance therapy for ulcerative colitis. N Engl J Med. 2013; 369(8):699-710.

14 Sandborn WJ, Feagan BG, Rutgeerts P, Hanauer S, Colombel JF, Sands BE, et al. Vedolizumab as induction and maintenance therapy for crohn's disease. N Engl J Med. 2013;369(8):711-21.

15 Vermeire S, Lukáš M, Magro F, Adsul S, Lindner D, Rosario M, et al. Vedolizumab efficacy, safety, and pharmacokinetics with reduced frequency of dosing from every 4 weeks to every 8 weeks in patients with crohn's disease or ulcerative colitis. J Crohns Colitis. 2020;14(8): 1066-73.

16 Ng SC, Hilmi IN, Blake A, Bhayat F, Adsul S, Khan QR, et al. Low frequency of opportunistic infections in patients receiving vedolizum$\mathrm{ab}$ in clinical trials and post-marketing setting. Inflamm Bowel Dis. 2018;24(11):243141.

17 Loftus EV Jr, Colombel JF, Feagan BG, Vermeire S, Sandborn WJ, Sands BE, et al. Longterm efficacy of vedolizumab for ulcerative colitis. J Crohns Colitis. 2016;11(4):400-11.

18 Winterfield LS, Menter A. Infliximab. Dermatol Ther. 2004;17(5):409-26.

19 Billmeier U, Dieterich W, Neurath MF, Atreya R. Molecular mechanism of action of anti-tumor necrosis factor antibodies in inflammatory bowel diseases. World J Gastroenterol. 2016;22(42):9300-13.

20 Wyant T, Leach T, Sankoh S, Wang Y, Paolino J, Pasetti MF, et al. Vedolizumab affects antibody responses to immunisation selectively in the gastrointestinal tract: randomised controlled trial results. Gut. 2015;64(1):77-83.
21 Rosario M, Dirks NL, Milch C, Parikh A, Bargfrede M, Wyant T, et al. A review of the clinical pharmacokinetics, pharmacodynamics, and immunogenicity of vedolizumab. Clin Pharmacokinet. 2017;56(11):1287-301.

22 Soler D, Chapman T, Yang LL, Wyant T, Egan $\mathrm{R}$, Fedyk ER. The binding specificity and selective antagonism of vedolizumab, an antialpha4beta7 integrin therapeutic antibody in development for inflammatory bowel diseases. J Pharmacol Exp Ther. 2009;330(3):86475.

23 Wyant T, Yang L, Fedyk E. In vitro assessment of the effects of vedolizumab binding on peripheral blood lymphocytes. MAbs. 2013; 5(6):842-50.

24 Neal RC, Ferdinand KC, Ycas J, Miller E. Relationship of ethnic origin, gender, and age to blood creatine kinase levels. Am J Med. 2009; 122(1):73-8.

25 Wong ET, Cobb C, Umehara MK, Wolff GA, Haywood LJ, Greenberg T, et al. Heterogeneity of serum creatine kinase activity among racial and gender groups of the population. Am J Clin Pathol. 1983;79(5):582-6.

26 Garcia W. Elevated creatine phosphokinase levels associated with large muscle mass: another pitfall in evaluating clinical significance of total serum CPK activity. JAMA. 1974; 228(11):1395-6.

27 Keltz E, Khan FY, Mann G. Rhabdomyolysis. The role of diagnostic and prognostic factors. Muscles Ligaments Tendons J. 2014;3(4): 303-12.

28 Sipponen T. Diagnostics and prognostics of inflammatory bowel disease with fecal neutrophil-derived biomarkers calprotectin and lactoferrin. Dig Dis. 2013;31(3-4):336-44.

29 Costa F, Mumolo MG, Bellini M, Romano MR, Ceccarelli L, Arpe P, et al. Role of faecal calprotectin as non-invasive marker of intestinal inflammation. Dig Liver Dis. 2003;35(9): 642-7.

30 Sands BE, Peyrin-Biroulet L, Loftus EV, Danese S, Colombel JF, Törüner M, et al. Vedolizumab versus adalimumab for moderateto-severe ulcerative colitis. N Engl J Med. 2019;381(13):1215-26. 